PROCEEDINGS OF THE AMERICAN MATHEMATICAL SOCIETY

Volume 124, Number 12, December 1996, Pages 3835-3841

S 0002-9939(96)03547-2

\title{
BANDS IN LATTICES OF OPERATORS
}

\author{
C. B. HUIJSMANS AND A. W. WICKSTEAD \\ (Communicated by Palle E. T. Jorgensen)
}

\begin{abstract}
We consider the lattice of regular operators on a Dedekind complete Banach lattice. We show that in general the projection onto a band generated by a lattice homomorphism need not be continuous and that the principal bands need not be closed for the operator norm. In fact it is possible to find a convergent sequence of operators all the members of which are disjoint from the limit.
\end{abstract}

\section{INTRODUCTION}

If $E$ and $F$ are Banach lattices, then it is possible to introduce an order structure in the space of norm bounded linear operators from $E$ into $F$ by defining $S \geq T$ to mean that $S x \geq T x$ whenever $x \geq 0$. There are many deficiencies of this order structure in general. It is rare that the order is generating, so attention is normally restricted to the regular operators, $\mathfrak{L}^{r}(E, F)$, the linear span of the cone of positive operators, i.e. those $S$ with $S \geq 0$. Even then the order structure is not often a lattice ordering. One of the few widely applicable cases where $\mathfrak{L}^{r}(E, F)$ is known to be a lattice is when $F$ is Dedekind complete, when $\mathfrak{L}^{r}(E, F)$ is also known to be a Dedekind complete vector lattice under this operator ordering.

It is known that $\mathfrak{L}^{r}(E, F)$ is, in this case, a Banach lattice under the so-called regular norm, defined by $\|T\|_{r}=\||T|\|$, where $\|\cdot\|$ denotes the usual operator norm, see e.g. [3], Theorem 15.2. However it is rare that the regular norm is the same as or even equivalent to the operator norm. It is, for example, if $F$ is an AM-space or if $E$ is an AL-space and $F$ has a Levi norm but not in general. By way of example, consider $E=F$, the Dedekind complete Banach lattice introduced in [7] by Krengel which we use below in Example 2.7. The operators $\tilde{S}_{n}$ used there have the property that $\left\|\tilde{S}_{n}\right\|=2^{-n / 2}$ whilst $\left\|\tilde{S}_{n}\right\|_{r}=1$ so it is impossible that the two norms are even equivalent. A similar argument can be applied to Example 2.4 below. Hence there is no reason to expect a nicely behaved interaction between the order structure in $\mathfrak{L}^{r}(E, F)$ and the operator norm. For example the lattice operations are not in general continuous. It came, therefore, as somewhat of a surprise when Voigt in [9] showed that if $E=F$, then the band projection of $\mathfrak{L}^{r}(E)$ onto its centre, which is simply the band generated by the identity operator, is not only continuous but

Received by the editors April 19, 1995 and, in revised form, June 26, 1995.

1991 Mathematics Subject Classification. Primary 47B65, 46B42.

Key words and phrases. Banach lattices, positive operators, bands, norm-closed.

This work was done whilst the second author was visiting Rijks Universiteit Leiden in the Summer of 1994 under the auspices of British Council/NWO Joint Scientific Research Project JRP131. 
is actually a contraction. A related result was proved (using techniques pioneered by Voigt) by Huijsmans and de Pagter in [6] where they showed that the band projection onto the band in $\mathfrak{L}^{r}(E, F)$ generated by a lattice homomorphism is also contractive, provided $F$ has a Fatou norm. They left open the question of whether or not this assumption on the norm could be omitted as it could be in Voigt's result. In $\S 2$ we show that it cannot be, although it can be weakened if one only requires continuity of the band projection.

If band projections are continuous, then, of course, their images and kernels are closed for the operator norm. The converse is not true, since $\mathfrak{L}^{r}(E)$ is not in general complete for the operator norm so the Closed Graph Theorem is not applicable. The examples we have mentioned so far leave open the possibility (see Question 3.3) that for an arbitrary lattice homomorphism $T$, the band $\{T\}^{d d}$ generated by $T$ and its complementary band, $\{T\}^{d}$, are closed. We show in $\S 3$ that this is certainly not the case for bands generated by a positive operator which is not assumed to be a lattice homomorphism. In fact we can show even more. It is possible to find a non-zero positive operator $T$ and a sequence $T_{n}$ in $\{T\}^{d}$ such that $T_{n} \rightarrow T$. This answers a problem posed by Abramovich [2]. Voigt's theorem shows, of course, that we cannot take $T$ to be the identity here.

We refer the reader to [3] or [8] for any unexplained terms in the theory of Banach lattices or positive operators.

\section{Continuity of the Band PRojection}

We need to use some notation that is not yet standardised in the literature, so we will spell out our exact definitions. The norm in a Dedekind complete Banach lattice is Fatou if $0 \leq x_{\gamma} \uparrow x \Rightarrow\left\|x_{\gamma}\right\| \uparrow\|x\|$. Equivalently, if $x_{\gamma}$ converges to $x$ in order (i.e. there is a net $y_{\gamma}$ directed downwards to zero with $\left|x_{\gamma}-x\right| \leq y_{\gamma}$ ), then $\|x\| \leq \liminf \left\|x_{\gamma}\right\|$. Fatou norms are also called lower semi-continuous norms in, for example, [6]. The norm is weakly Fatou if there is a constant $m \geq 1$ such that $0 \leq x_{\gamma} \uparrow x \Rightarrow\|x\| \leq m \sup _{\gamma}\left\|x_{\gamma}\right\|$; see [10], §107. Obviously every order continuous norm is Fatou and every Fatou norm is weakly Fatou.

Examples 2.1. If $S$ is an infinite Stonean space, then the norm in $C(S)$ is not order continuous although the norm is clearly Fatou.

Let $S$ be a Stonean space and $s_{0}$ a non-isolated point of $S$. Fix $\alpha>0$ and let $\|\cdot\|_{\alpha}$ denote the norm on $C(S)$ defined by $\|f\|_{\alpha}=\|f\|_{\infty}+\alpha\left|f\left(s_{0}\right)\right|$, where $\|\cdot\|_{\infty}$ denotes the usual supremum norm on $C(S)$. The constantly one function 1 has norm $1+\alpha$ but the upward directed set of those functions lying between the zero function $\mathbf{0}$ and $\mathbf{1}$ and which vanish at $s_{0}$ has supremum equal to $\mathbf{1}$. All these functions have norm at most 1 , showing that the $\|\cdot\|_{\alpha}$ norm on $C(S)$ is not Fatou. It is simple to see that it is weakly Fatou, where we can take $m=1+\alpha$.

Let $E_{m}$ denote $C(S)$ equipped with $\|\cdot\|_{m}$ and consider $\ell_{\infty}\left(E_{m}\right)=\left\{\left(f_{m}\right): f_{m} \in\right.$ $E_{m}$ and $\left.\left(\left\|f_{m}\right\|_{m}\right) \in \ell_{\infty}\right\}$ normed by $\left\|\left(f_{m}\right)\right\|=\sup \left\{\left\|f_{m}\right\|_{m}: m \in \mathbb{N}\right\}$ and ordered by the pointwise partial order. This is a Dedekind complete Banach lattice. The norm in this is clearly not weakly Fatou. Examples like this have been used many times before. For example, Abramovich uses it in Example 5 of [1] and refers there to an earlier use by Veksler, which was possibly the first use of it.

If $E$ is Dedekind complete with a Fatou norm and $T: E \rightarrow E$ is a lattice homomorphism, then the band projection onto the band generated by $T$ is contractive, 
by Theorem 5.5 of [6]. The condition on the norm cannot be weakened to weakly Fatou as the following example shows.

Example 2.2. In Examples 2.1, choose $S$ and $s_{0}$ so that there is a homeomorphism $\phi$ of $S$ onto itself with $\phi s_{0} \neq s_{0}$. Fix a constant $\alpha>0$ and equip $C(S)$ with the corresponding norm. Define two operators $T, U: C(S) \rightarrow C(S)$ by

$$
\begin{aligned}
& T f(s)=f\left(\phi s_{0}\right) \mathbf{1}, \\
& U f(s)=f(\phi s) .
\end{aligned}
$$

Note that both $T$ and $U$ are lattice homomorphisms and thus are certainly positive.

We claim that $T$ and $U$ are disjoint in the lattice of regular operators on $C(S)$. Indeed, if $0 \leq V \leq T, U$, then for each $f \in C(S)_{+}$and $s \neq s_{0}$ we may find a clopen set $A$ with $\phi s_{0} \in A, \phi s \in S \backslash A$. If $g=f \chi_{A}$ and $h=f\left(\mathbf{1}-\chi_{A}\right)=f \chi_{S \backslash A}$, then $f=g+h, g, h \geq 0$ and $g \perp h$. Thus we have

$$
\begin{aligned}
0 & \leq V g(s) \\
& \leq U g(s) \\
& =g(\phi s) \\
& =f(\phi s) \chi_{A}(\phi s) \\
& =0
\end{aligned}
$$

and

$$
\begin{aligned}
0 & \leq V h(s) \\
& \leq T h(s) \\
& =h\left(\phi s_{0}\right) \mathbf{1} \\
& =f\left(\phi s_{0}\right) \chi_{S \backslash A}\left(\phi s_{0}\right) \\
& =0 .
\end{aligned}
$$

Thus

$$
V f(s)=V g(s)+V h(s)=0+0=0
$$

whenever $f \geq 0$ and $s \neq s_{0}$. As $s_{0}$ is not isolated in $S$ we see that $V f$ is the zero function for all positive $f$ and hence for all $f \in C(S)$.

Note that $((U-T) f)\left(s_{0}\right)=0$, so that $\|(U-T) f\|_{\alpha}=\|(U-T) f\|_{\infty}$. For each $s \in S$ we have

$$
\begin{aligned}
|U f(s)-T f(s)| & =\left|f(\phi s)-f\left(\phi s_{0}\right)\right| \\
& \leq|f(\phi s)|+\left|f\left(\phi s_{0}\right)\right| \\
& \leq 2\|f\|_{\infty} \\
& \leq 2\|f\|_{\alpha}
\end{aligned}
$$

so that we have $\|(U-T) f\|_{\alpha} \leq 2\|f\|_{\alpha}$ for all $f \in C(S)$ and hence $\|U-T\| \leq 2$ given that $C(S)$ is equipped with the norm $\|\cdot\|_{\alpha}$.

Use Urysohn's lemma to find $h \in C(S)$ with $\mathbf{0} \leq h \leq 1, h\left(\phi s_{0}\right)=1$ and $h\left(s_{0}\right)=$ 0 , so that

$$
\|h\|_{\alpha}=\|h\|_{\infty}+\alpha\left|h\left(s_{0}\right)\right|=\|h\|_{\infty}=1
$$

but $T h(s)=h\left(\phi s_{0}\right)=1$ for all $s \in S$, so that $\|T h\|_{\alpha}=1+\alpha$. It follows that $\|T\| \geq 1+\alpha$. In a similar manner we see that $\|U\| \geq 1+\alpha$. 
Whether we consider the band projection of the space of regular operators on $C(S)$ onto the band generated by $T$ or by $U$, by looking at the image of $U-T$ we see that this projection has norm at least $(1+\alpha) / 2$. Taking $\alpha>1$ shows that the projection need not be contractive.

A close inspection of the proof of Theorem 5.5 in [6] shows that we have the following result.

Theorem 2.3. Let $E$ be a Dedekind complete Banach lattice with a weakly Fatou norm and let $T$ be a lattice homomorphism on $E$. The band projection of $\mathfrak{L}^{r}(E)$ onto $\{T\}^{d d}$ is continuous with respect to the operator norm.

Again the weak Fatou property of the norm may not be omitted from the statement of this theorem.

Example 2.4. With the notation of Examples 2.1, let $E=\ell_{\infty}\left(E_{m}\right)$. Let $U_{m}, T_{m}$ denote the operator on $E_{m}$ constructed as in Example 2.2 taking $\alpha=m$. Define $T$ on $E$ by

$$
T\left(f_{m}\right)=\left(\frac{T_{m} f_{m}}{\left\|T_{m}\right\|}\right)
$$

so that $\|T\|=1$. It is routine to verify that $T$ is a lattice homomorphism.

Let $\tilde{U}_{n}$ denote the operator on $E$ taking $\left(f_{m}\right)$ to the sequence in $E$ all of whose terms are zero apart from the $n$th which is $U_{n} f_{n}$ with $\tilde{T}_{n}$ defined similarly. Clearly $\tilde{U}_{n} \perp T$ for all $n \in \mathbb{N}$ and we also have $\tilde{T}_{n} \in\{T\}^{d d}$ as $0 \leq \tilde{T}_{n} \leq\left\|T_{n}\right\| T$. If $P$ denotes the band projection of $\mathfrak{L}^{r}(E)$ onto the band generated by $T$, then we see that $\left\|\tilde{U}_{n}-\tilde{T}_{n}\right\| \leq 2$ whilst

$$
\left\|P\left(\tilde{U}_{n}-\tilde{T}_{n}\right)\right\|=\left\|\tilde{T}_{n}\right\|=\left\|T_{n}\right\| \geq n+1
$$

so that $P$ is not norm bounded and therefore not continuous.

What, then, is so special about the band generated by the identity? As our next result shows, the invertibility of the identity operator is the crucial thing here, as long as we are looking only for continuity of the band projection. Notice that the ideas in the following proof show that, as is the case with the centre, the band generated by a lattice isomorphism is simply the ideal that it generates.

Theorem 2.5. Let $E$ be any Dedekind complete Banach lattice, let $S$ be a lattice isomorphism on $E$ and let $T: E \rightarrow E$ be such that $-S \leq T \leq S$. The band projection of $\mathfrak{L}^{r}(E)$ onto $\{T\}^{d d}$ is continuous with respect to the operator norm.

Proof. The operator, $L_{S}$, of left multiplication by $T$ is an order isomorphism on $\mathfrak{L}^{r}(E)$ with inverse $L_{S^{-1}}$. The image of the centre of $E$ under $L_{S}$ is the band generated by $S$. If $U \in \mathfrak{L}^{r}(E)$, then the band projection of $U$ onto the band generated by $T$ may be calculated by forming $L_{S^{-1}}(U)$, projecting that onto the centre of $E$, then onto the band generated by $L_{S^{-1}}(T)$ in the centre of $E$ and then mapping this image back using $L_{S}$. I.e. if $P_{T}$ denotes the projection onto the band generated by $T, P_{I}$ that onto the band generated by the identity and $Q$ that of the centre of $E$ onto the band generated by $L_{S^{-1}}(T)$, then

$$
P_{T}=L_{S} \circ Q \circ P_{I} \circ L_{T^{-1}}
$$

so that we have

$$
\left\|P_{T}\right\| \leq\left\|L_{S}\right\|\|Q\|\left\|P_{I}\right\|\left\|L_{S^{-1}}\right\|=\|S\|\left\|S^{-1}\right\|
$$


(noting that order isomorphisms on Banach lattices are automatically continuous) and we see, in particular, that $P_{T}$ is continuous.

Notice that, in the terminology of Arendt and Hart [4], the lattice homomorphism $T$ constructed in Example 2.4 is quasi-invertible so that we cannot weaken invertibility in Theorem 2.5 to quasi-invertibility.

An alternative direction in which we might seek to extend the results of Voigt and Huijsmans-de Pagter is by widening the class of operators at the expense of strengthened hypotheses on the Banach lattice $E$. In the paper [6] the authors consider the ideal and the band generated by the lattice homomorphisms. The positive operators in the ideal generated by the lattice homomorphisms are simply the finite sums of lattice homomorphisms and have been characterised in an alternative manner in [5]. Even for the case of an operator in the ideal generated by the lattice homomorphisms on finite dimensional Banach lattices (where the norm is as nice as might be desired) the corresponding band projection need not be a contraction.

In order to see this we refer to Krengel's examples in [7], where he shows that on $2^{n}$-dimensional Euclidean space $\ell_{2}^{2^{n}}$ there is an operator $S_{n}$ such that $\left\|S_{n}\right\|=2^{-n / 2}$ and $\left\|\left|S_{n}\right|\right\|=1$. Using the standard basis and ordering on finite dimensional spaces, any matrix with only one non-zero positive entry represents a lattice homomorphism, so that all operators on finite dimensional Euclidean space are in the band generated by the lattice homomorphisms. In particular $S_{n}^{ \pm}$are in this band. The band projection of $S_{n}$ onto the band generated by either of $S_{n}^{ \pm}$is the operator generating the band, and one of these must have norm at least $1 / 2$ so that the corresponding band projection has norm at least $2^{n / 2-1}$ and so is not a contraction if $n>2$.

We do however have continuity of the projection in such cases assuming only that the norm on $E$ is weakly Fatou.

Proposition 2.6. Let $E$ be a Dedekind complete Banach lattice with a weakly Fatou norm and let $T$ be a finite sum of lattice homomorphisms on $E$. The band projection of $\mathfrak{L}^{r}(E)$ onto $\{T\}^{d d}$ is continuous with respect to the operator norm.

Proof. Let $T=\sum_{k=1}^{n} T_{k}$, where each $T_{k}$ is a lattice homomorphism, so that $\{T\}^{d}=$ $\left\{T_{1}, T_{2}, \ldots, T_{n}\right\}^{d}=\bigcap_{k=1}^{n}\left\{T_{k}\right\}^{d}$. To establish the results it suffices to prove that the band projection onto $\{T\}^{d}$ is operator norm continuous. But this is the composition of the $n$ band projections onto $\left\{T_{k}\right\}^{d}$, each of which is continuous by Theorem 2.3, so is certainly continuous.

However it does not seem possible to find sufficiently strong conditions on $E$ (other than being finite dimensional) which will suffice to force continuity of the band projection onto $\{T\}^{d d}$ even if we consider only operators $T$ from the closed ideal generated by the lattice homomorphisms.

Example 2.7. Take for $E$ the $\ell_{2}$-sum of $\ell_{2}\left(2^{n}\right)$ for $n \in \mathbb{N}$, so that this is norm and order isomorphic to $\ell_{2}$ with its usual norm and order. By, if necessary, replacing $S_{n}$ by $-S_{n}$ we may suppose that $T_{n}=S_{n}^{+}$has norm at least $1 / 2$, and define $T$ on $E$ by $T\left(x_{n}\right)=\left(T_{n} x_{n} / n\right)$ where $x_{n} \in \ell_{2}\left(2^{n}\right)$. Each $T_{n}$ is a finite sum of lattice homomorphisms, so we see that $T$ is certainly in the norm closure of the ideal of finite sums of lattice homomorphisms on $E$. If we define $\tilde{T}_{n}$ as we did in Example 2.4 and $\tilde{S}_{n}$ analogously, then we see that the band projection onto $T$ of $\tilde{S}_{n}$ is $\tilde{T}_{n}$. But $\left\|\tilde{T}_{n}\right\|=\left\|T_{n}\right\| \geq 1 / 2$ whilst $\left\|\tilde{S}_{n}\right\|=\left\|S_{n}\right\|=2^{-n / 2}$ so that this band projection cannot be norm bounded. 


\section{WHEN ARE BANDS CLOSED?}

As we pointed out earlier, it is conceivable that both a band in $\mathfrak{L}^{r}(E)$ and its complementary band are norm closed without the corresponding band projection being continuous for the operator norm. The examples that we have discussed above do not give examples where a band must fail to be closed. In the last example, for instance, it is simple to see that both the band generated by $T$ and its complementary band are norm closed.

Although one should not normally expect bands in $\mathfrak{L}^{r}(E)$ to be norm closed, it follows from results above that it does happen in some cases. It is tempting therefore to hope that at least the more outrageous kind of behaviour that may seem a priori possible might not happen. For example, Abramovich asked in [2] whether the facts that $T_{n} \perp T$ and $T_{n} \rightarrow T$ were enough to force $T=0$. This is true if $T$ is a lattice homomorphism and either $T$ is invertible or $E$ has a weakly Fatou norm, but in general it is possible to have $T \neq 0$. Our intuition derived from study of Banach lattices is not at all a good guide to the behaviour of lattices of operators on Banach lattices!

Example 3.1. For each $n \in \mathbb{N}$ let $\mu_{n}$ denote the probability measure on the collection $\Sigma_{n}$ of all subsets of a $2^{n}$-element set $S_{n}$ which assigns weight $2^{-n}$ to each singleton. Write $S$ for the countable product $\prod S_{n}$ and let $\mu$ denote the corresponding product measure on the product $\sigma$-algebra $\Sigma$. Our example will act on the Banach lattice $L^{2}(\mu)$, which is almost as well-behaved a Banach lattice as one might hope for. This space may be identified with a (countably) infinite Hilbert space tensor product of finite dimensional spaces which are isometrically order isomorphic to $\ell_{2}\left(2^{n}\right)$.

We use the operators $S_{n}$ and $T_{n}$ introduced in Example 2.7, and set $U_{n}=$ $S_{n} /\left\|T_{n}\right\|$ and $V_{n}=T_{n} /\left\|T_{n}\right\|$, so that $V_{n}=U_{n}^{+},\left\|V_{n}\right\|=1$ and $\left\|U_{n}\right\| \leq 2^{1-n / 2}$. Using the tensor product representation of $L^{2}(\mu)$, we may form the product operator $P=\bigotimes_{n=1}^{\infty} V_{n}$ on $L^{2}(\mu)$ which has norm $\prod_{n=1}^{\infty}\left\|V_{n}\right\|=1$. For each $n \in \mathbb{N}$ we may also form the operator

$$
Q_{n}=\left(\bigotimes_{k=1}^{n-1} V_{n}\right) \otimes U_{n}^{-} \otimes\left(\bigotimes_{k=n+1}^{\infty} V_{n}\right)
$$

which also acts on $L^{2}(\mu)$ and has norm $\left\|U_{n}^{-}\right\|$. It is relatively routine, if slightly tedious, to write out in full that $Q_{n}$ is disjoint from $P$ for each $n \in \mathbb{N}$. In tensor product notation, we may write

$$
P-Q_{n}=\left(\bigotimes_{k=1}^{n-1} V_{n}\right) \otimes U_{n} \otimes\left(\bigotimes_{k=n+1}^{\infty} V_{n}\right)
$$

showing that it has norm equal to $\left\|U_{n}\right\|$. It follows that even though $Q_{n} \perp P$ for each $n \in \mathbb{N}$, we have $Q_{n} \rightarrow P$, yet $\|P\|=1$, showing that $P \neq 0$. It follows that the complementary band, $\{P\}^{d}$, is not closed in the case, as $P$ lies in its closure!

It is not too difficult to see that the band $\{P\}^{d d}$ is closed. However the sum $\sum_{n=1}^{\infty} Q_{n} / 2^{n}$ is norm convergent to a limit $Q$. The fact that the positive cone in $\mathfrak{L}^{r}(E)$ is closed under the operator norm suffices to show that $\sum_{n=1}^{\infty} Q_{n} / 2^{n} \geq$ $Q_{m} / 2^{m} \geq 0$ for each $m \in \mathbb{N}$, so that $Q_{m} \in\{Q\}^{d d}$ even though $Q_{m} \rightarrow P \in\{Q\}^{d}$. Thus even principal bands in $\mathfrak{L}^{r}\left(L^{2}(\mu)\right)$ need not be norm closed.

Our examples leave open the following two problems. 
Question 3.2. If $E$ is a Dedekind complete Banach lattice, then arguments similar to those used in Theorem 2.5 and Proposition 2.6 show that the projection onto the band (=ideal) generated by a finite sum of lattice isomorphisms is continuous. A little more work shows that this is also true for operators in the ideal generated by them. Is this true for operators in the band generated by the lattice isomorphisms?

Question 3.3. If $E$ is a Dedekind complete Banach lattice and $T$ a lattice homomorphism on $E$, must $\{T\}^{d}$ and $\{T\}^{d d}$ both be operator norm closed in $\mathfrak{L}^{r}(E)$ ?

\section{ADDED IN PROOF}

Z. L. Chen has recently shown that both of these questions have negative answers.

\section{REFERENCES}

1. Y. A. Abramovich, On the maximal normed extension of the semi-ordered spaces, Vestnik Leningr. Univ. Mat. Meh. Astronom. 1970, no. 1, 7-17; English transl., Vestnik Leningrad Univ. Math. 3 (1976), 1-12. MR 43:3767

2. Y. A. Abramovich, Private Communication.

3. C. D. Aliprantis and O. Burkinshaw, Positive Operators, Academic Press, New York \& London, 1985. MR 87h:47086

4. W. Arendt and D. R. Hart, The spectrum of quasi-invertible disjointness preserving operators, J. Funct. Anal. 68 (1986), 149-167. MR 87j:47051

5. S. J. Bernau, C. B.Huijsmans and B. de Pagter, Sums of lattice homomorphisms, Proc. Amer. Math. Soc. 115 (1992), 151-156. MR 92h:46009

6. C. B. Huijsmans and B. de Pagter, Disjointness preserving and diffuse operators, Compositio Math. 79 (1991), 351-374. MR 92k:47071

7. U. Krengel, Remark on the modulus of compact operators, Bull. Amer. Math. Soc. 72 (1966), 132-133. MR 32:8162

8. P. Meyer-Nieberg, Banach Lattices, Springer-Verlag, Berlin Heidelberg New York, 1991. MR 93f: 46025

9. J. Voigt, The projection onto the center of operators in a Banach lattice, Math. Z. 199 (1988), 115-117. MR 89f: 47058

10. A. C. Zaanen, Riesz Spaces II, North-Holland, Amsterdam, New York, London, 1983. MR 86b: 46001

Mathematisch Instituut, Rijksuniversiteit Leiden, Niels Bohrweg 1, 2333 CA Leiden, The Netherlands

E-mail address: chuijsmans@rulcri.leidenuniv.nl

Department of Pure Mathematics, The Queen's University of Belfast, Belfast BT7 $1 \mathrm{NN}$, NoRTHERn IRELAND

E-mail address: a.wickstead@qub.ac.uk 